HETEROCYCLES, Vol. , No. , , pp. -. (c) The Japan Institute of Heterocyclic Chemistry

Received, Accepted, Published online, COM-06-(Please do not delete.)

\title{
AN EFFICIENT SYNTHESIS OF PROCYANIDINS USING EQUIMOLAR CONDENSATION OF CATECHIN AND/OR EPICATECHIN CATALYZED BY YTTERBIUM TRIFLATE
}

\section{Yoshihiro Mohri, ${ }^{a}$ Masayoshi Sagehashi, ${ }^{\text {a }}$ Taiji Yamada, ${ }^{a}$ Yasunao Hattori,, ${ }^{b, c}$ Keiji Morimura, ${ }^{\text {b,d }}$ Yasunori Hamauzu, ${ }^{\text {a }}$ Tsunashi Kamo, Mitsuru Hirota, ${ }^{e}$ and Hidefumi Makabe ${ }^{\mathrm{a} *}$}

${ }^{\mathrm{a}}$ Sciences of Functional Foods, Graduate School of Agriculture, Shinshu University, 8304 Minami-minowa, Kami-ina, Nagano 399-4598, Japan $\quad$ b Interdisciplinary Graduate School of Science and Technology, Shinshu University, 8304 Minami-minowa, Kami-ina, Nagano 399-4598, Japan $\quad$ 'Satellite Venture Business Laboratory, Shinshu University, 3-15-1 Tokida, Ueda, Nagano 386-8567, Japan ${ }^{\mathrm{d}}$ Geol Cosmetics. Co., LTD, 111-1 Shinmura, Katsuragi, Nara 639-2121, Japan $\quad{ }^{\mathrm{e}}$ Department of Bioscience and Biotechnology, Faculty of Agriculture, Shinshu University, 8304, Minami-minowa, Kami-ina, Nagano 399-4598, Japan * *E-mail address: makabeh@shinshu-u.ac.jp

\begin{abstract}
Stereoselective synthesis of catechin and epicatechin dimers under intermolecular condensation of equimolar amount of catechin derivatives catalyzed by $\mathrm{Yb}(\mathrm{OTf})_{3}$. The coupled products were successfully converted to procyanidin B1, B2, B3, and B4, respectively. Procyanidin B1, B2, B3, and B4 could be used as standard compounds for identifying the polyphenols in natural source.
\end{abstract}

\section{INTRODUCTION}

Proanthocyanidins are known as condensed or noncondensed hydrolysable tannins. ${ }^{1}$ These condensed tannins can be found in the vegetables kingdom. ${ }^{2}$ In particular, they exist in grape seeds and skins and red wines. Many biological activities, mainly a powerful free-radical scavenging activity, have been reported for flavonoids, and their investigation is increasingly important. Especially, procyanidins were paid

$\dagger$ Dedicated to the late Professor John W. Daly for his outstanding contribution to natural product chemistry. 
attention since the relationship between procyanidin content and vasoactive properties of red wine have been reported by Coder and co-workers in 2006. ${ }^{3}$ Tannin extracts from plants give various types of polyphenol. Because their identification as well as purification is extremely difficult, further studies of proanthocyanidins remains. Recently, to obtain procyanidin oligomers in pure state, synthetic efforts were devoted. ${ }^{4}$ However, efficient syntheses are very limited because the formation of the intermolecular C-4-C-8 bond has some problems. The typical synthetic methods are as follows. The first example is nucleophilic addition of C-8 lithiated nucleophile onto a C-4 protected ketocatechin as a substrate. ${ }^{5}$ This reaction generally proceeds with the regioselective and oligomerization control demands of the coupling reaction, however, it does not satisfy the stereochemical requirement of the newly formed C-4 asymmetric center. The next is the nucleophilic substitution method which needs to use nucleophilic partner in large excess (3.0-4.5 eq.) to prevent further oligomerization. Thus the efficient synthetic method to prepare procyanidin dimers has some restrictions, although recent advance was made in the regio and stereoselective reaction. ${ }^{4}$ Until now, only a few attempts to prepare procyanidin dimers under stoichiometric conditions have been reported in the literature. The first example is an intramolecular coupling of monomeric units bound by a temporary diester link. ${ }^{6}$ This method is suitable for synthesizing procyanidin B1 (1) and B3 (3), however, it suffers from low yield of condensation for synthesizing B2 (2) and B4 (4). The second is reported by E. Fouquet and co-workers. ${ }^{7}$ They synthesized procyanidin dimers based on the intermolecular nucleophilic substitution of C-4 activated and C-8 halogenated monomer to prevent further oligomerization using $\mathrm{TiCl}_{4}$ as a Lewis acid. This reaction needs large excess of $\mathrm{TiCl}_{4}$. Quite recently, Oyama and co-workers reported the efficient synthesis of procyanidin B3. They use 4-acetoxy-3- $O$-acetyl-perbenzylcatechin as an electrophile. They made success to reduce the amount of nucleophile up to 1.5 eq. ${ }^{4 \mathrm{e}}$ In the course of our research, we have developed a very simple and efficient intermolecular synthesis of procyanidin dimers. ${ }^{8}$ The key step is a coupling reaction between equimolar amounts of tetra-benzylated monomer 5a (nucleophile) and a C-4 activated monomer 6a (electrophile) using 1.0 eq. of rare earth metal Lewis acid such as $\mathrm{Yb}(\mathrm{OTf})_{3}$ (Figure. 1).

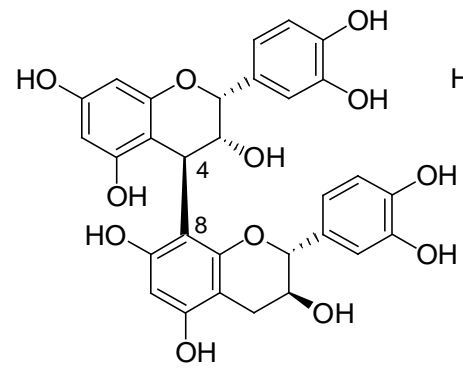

procyanidin B1 (1)

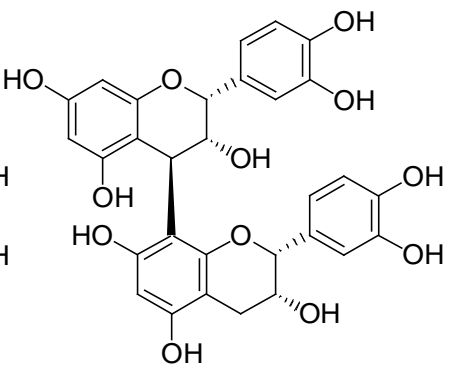

procyanidin B2 (2)

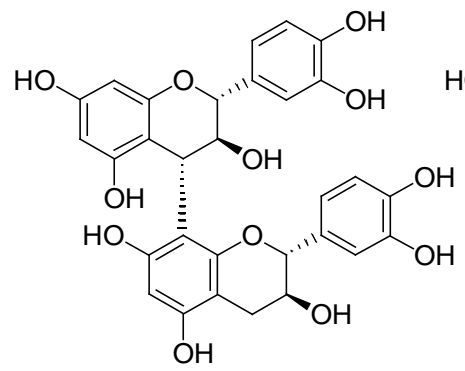

procyanidin B3 (3)

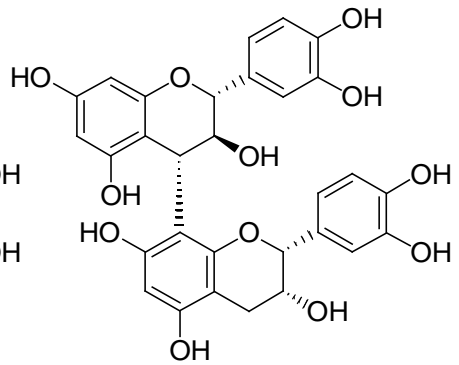

procyanidin B4 (4)

Figure 1. The structures of procyanidin B1-B4 (1-4). 


\section{RESULTS AND DISCUSSION}

We chose tetrabenzylated catechin 5a, a nucleophilic unit, prepared by the Kawamoto's procedure ${ }^{9}$ and electrophile unit 6a prepared by the Saito's method. ${ }^{10}$ Equimolar condensation of $5 \mathbf{a}$ and $\mathbf{6 a}$ at rt was examined using various Lewis acids including rare earth metal in $\mathrm{CH}_{2} \mathrm{Cl}_{2}$ (Table 1).

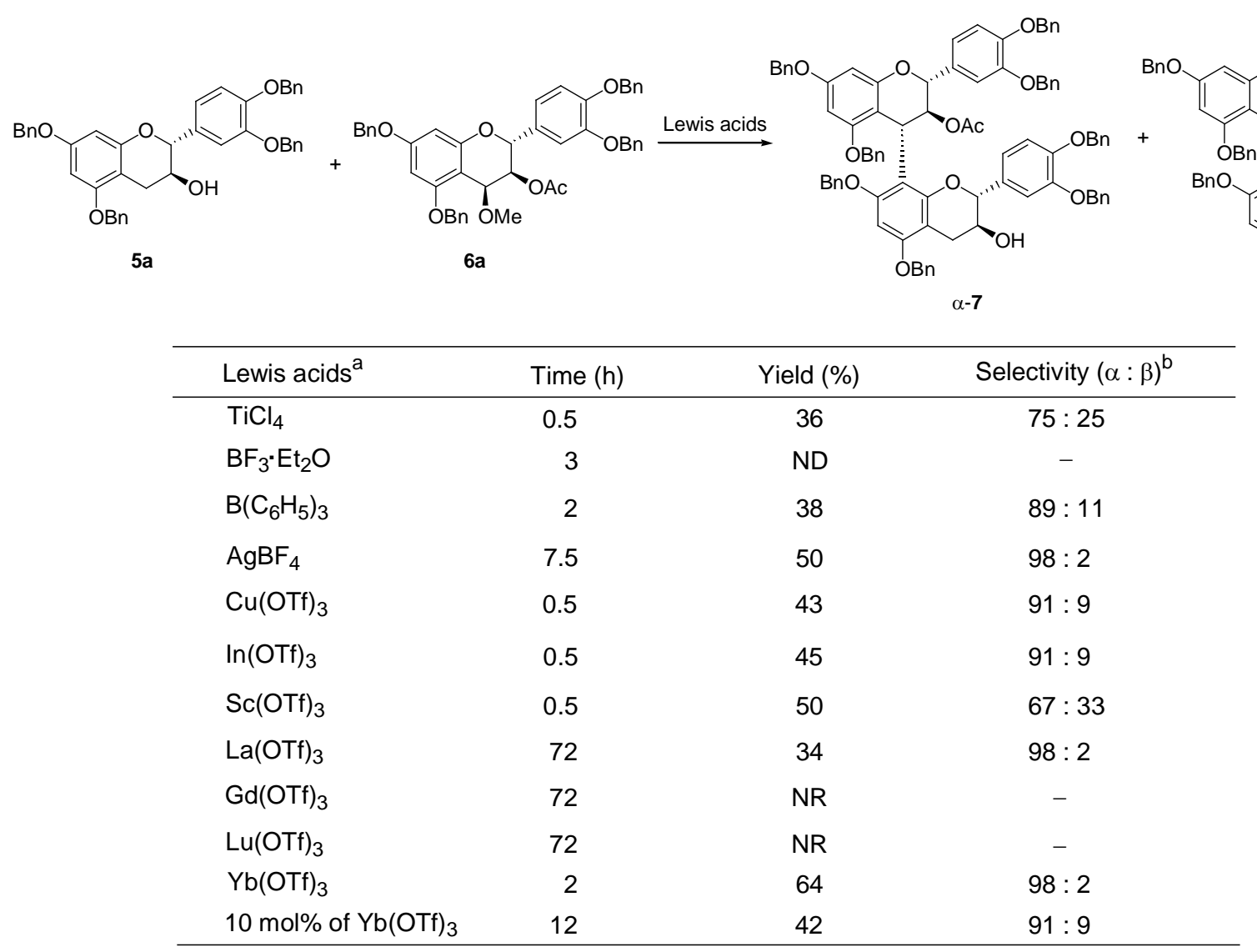

$\mathrm{a}_{1.0}$ equivalent of Lewis acid was used otherwise noted.

${ }^{\mathrm{b}}$ The selectivity was determined by ${ }^{1} \mathrm{H}$ NMR analysis of $\mathrm{C}-3$ position of diacetate derivative of $\alpha-7$ (5.80 and $5.83 \mathrm{ppm})$ and $\beta-7$ (5.53 and $5.58 \mathrm{ppm})$ according to the reported procedure. ${ }^{4 \mathrm{~b}}$

Table 1. Lewis acids mediated coupling reaction between $5 \mathbf{a}$ and $\mathbf{6 a}$

The first attempt at the coupling reaction was conducted with equimolar amounts of the protected catechin 5a and the acetylated substrate 6a to obtain $\alpha-7$, which is the precursor of procyanidin B3 (3). Typical Lewis acids, such as $\mathrm{TiCl}_{4}$ and $\mathrm{BF}_{3} \bullet \mathrm{Et}_{2} \mathrm{O}$ gave sluggish results. These reactions required a large excess of the nucleophile at low temperature in order to limit the reaction of the activated monomer with itself or with the dimeric product leading in both cases to oligomeric side products. ${ }^{9,10}$ The next attempt at the coupling reaction was conducted with late transition metals as Lewis acids. Among Ag, $\mathrm{Cu}$, and In, especially $\mathrm{AgBF}_{4}$ gave a good selectivity with moderate chemical yield. ${ }^{11}$ We further paid attention to rare metal Lewis acids such as Sc and La. While Sc gave poor stereoselectivity, La afforded high selectivity although the chemical yield was 34\%. This result encouraged us to replace La to Yb. The reaction furnished good selectivity with 64\% yield. When reaction time was longer than $0.5 \mathrm{~h}$, further condensed products were observed. The catalytic amount of $\mathrm{Yb}(\mathrm{OTf})_{3}(10 \mathrm{~mol} \%)$ also afforded coupled 
product in $42 \%$ yield at 91:9 ratio of the desired product. This result indicates that this reaction could be carried out using catalytic amount of $\mathrm{Yb}(\mathrm{OTf})_{3}$. Interestingly, $\mathrm{Gd}(\mathrm{OTf})_{3}$ and $\mathrm{Lu}(\mathrm{OTf})_{3}$ did not give any condensed product. The reported condensation reaction between catechin nucleophile 5a and catechin electrophile 6a required large amount excess of catechin nucleophile $\mathbf{5 a}$ to obtain desired dimer in high yield. $^{10,12}$ Using large excess amount of nucleophile is a big problem because composition of desired coupled product is only a small part in the reaction system and it is necessary to get rid of large amount of starting material. Optimized equimolar condensation is extremely important for an efficient synthesis of catechin dimers. Next, we examined the condensation of the combination of catechin nucleophile 5a and epicatechin nucleophile $\mathbf{5 b}$ with catechin electrophile $\mathbf{6 a}$ and/or epicatechin electrophile $\mathbf{6 b}$ using $\mathrm{Yb}(\mathrm{OTf})_{3}$ as a Lewis acid. In each case, the reaction worked well. As to the stereoselectivty, however, the epicatechin nucleophile $\mathbf{5 b}$ gave a little bit poor results compared to catechin nucleophile $\mathbf{5 a}$. In case of tri-benzylated phloroglucinol, the stereoselectivity of $\mathbf{1 1}$ showed 75:25 ratio. ${ }^{13}$ Some stereochemical requirement seems to be necessary to get high selectivity (Scheme 1).

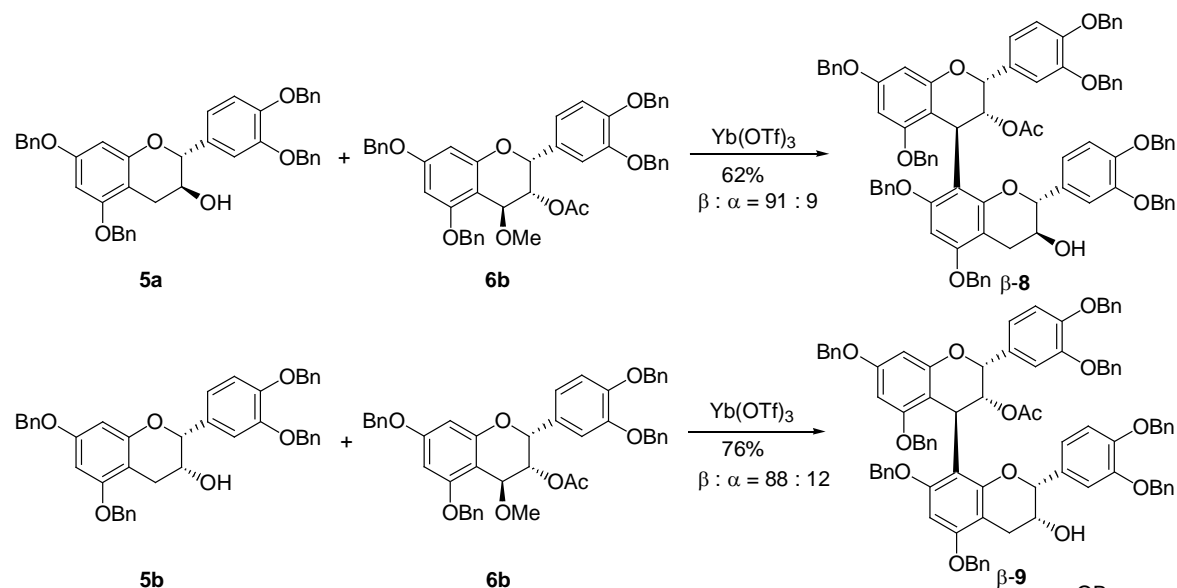

$5 b$

6

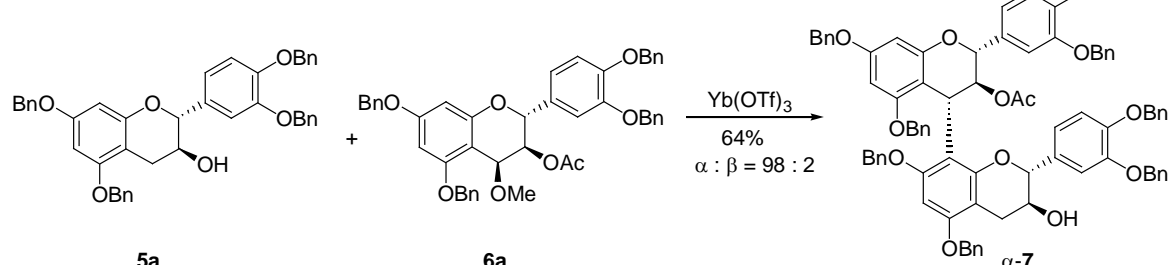

$5 a$

$6 a$

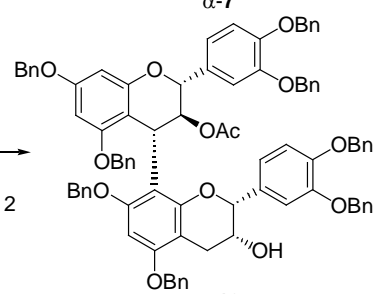

5b

$6 a$

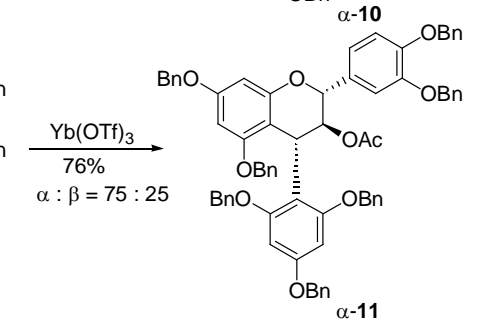

16

$6 a$

Scheme 1. Equimolar coupling of catechin and epicatechin using $\mathrm{Yb}(\mathrm{OTf})_{3}$ as a Lewis acid. 
Finally, condensed compounds $\alpha-\mathbf{7}, \beta-\mathbf{8}, \beta-\mathbf{9}$, and $\alpha-\mathbf{1 0}$ were subjected to the hydrolysis of the acetate with $\mathrm{K}_{2} \mathrm{CO}_{3}$ in $\mathrm{MeOH}$ followed by debenzylidation by $\mathrm{Pd}(\mathrm{OH})_{2}$ in THF-MeOH- $\mathrm{H}_{2} \mathrm{O}$ catalyzed hydrogenolysis to give procyanidin B1 (1)-B4 (4). All the spectral data for 1-4 were similar to those of the reported values (Scheme 3). ${ }^{4 \mathrm{a}, 4 \mathrm{~d}, 6 \mathrm{a}}$
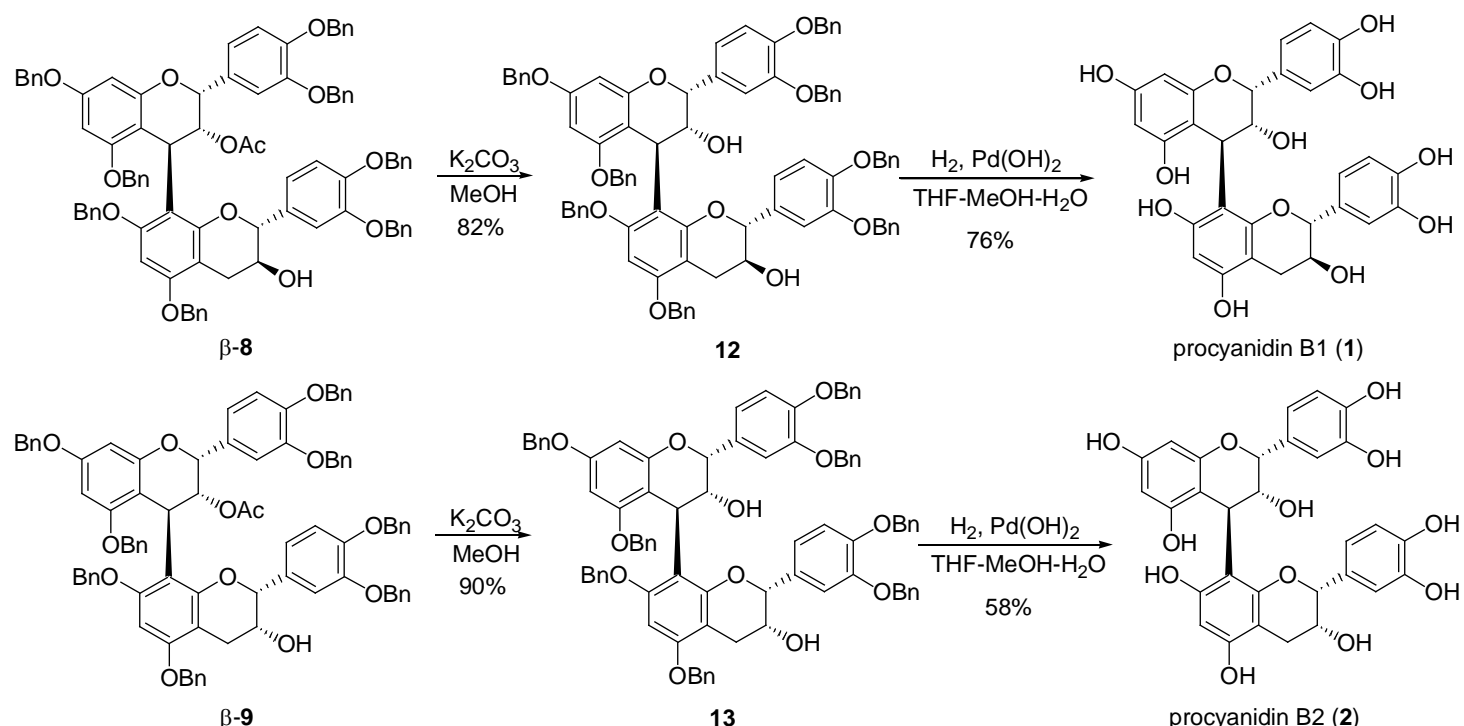

procyanidin B1 (1)
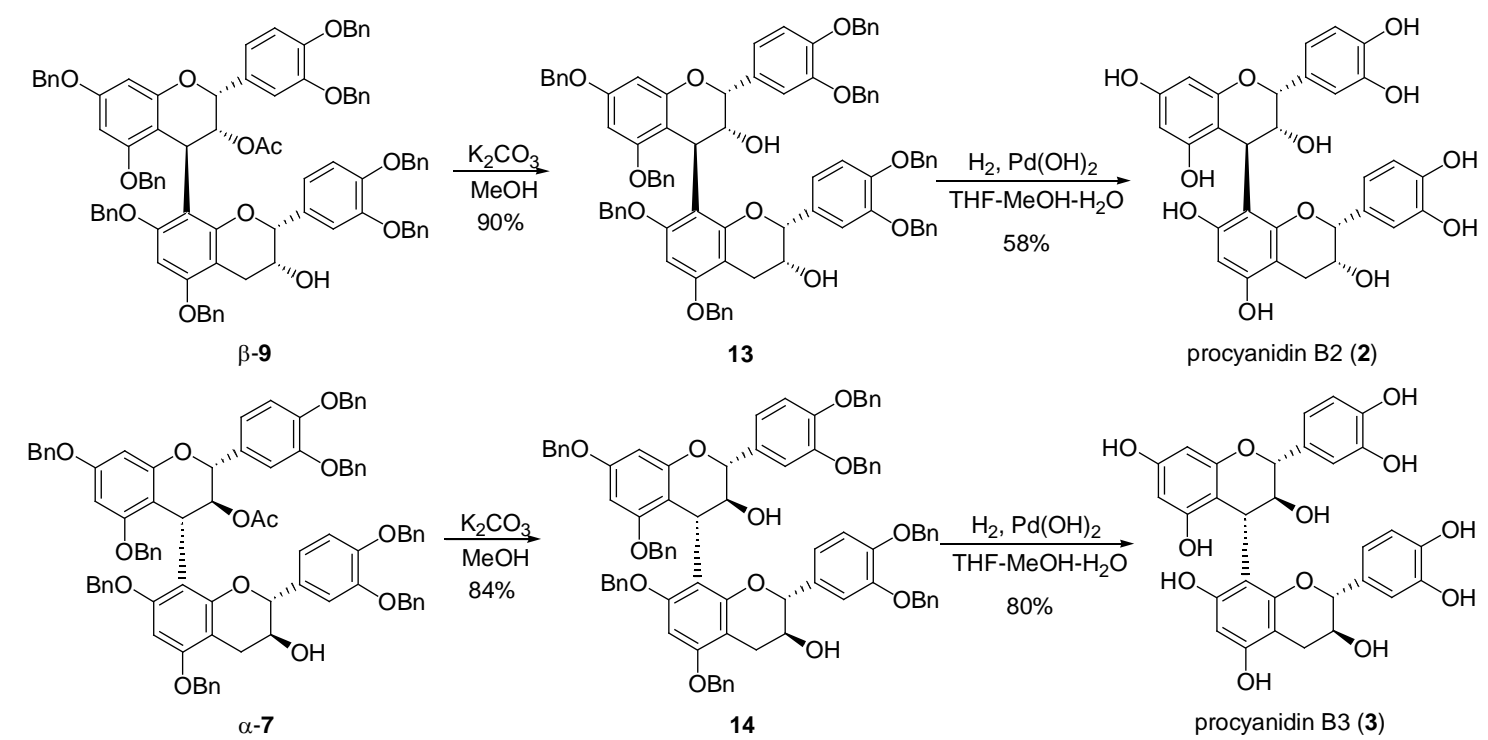

procyanidin B2 (2)

14
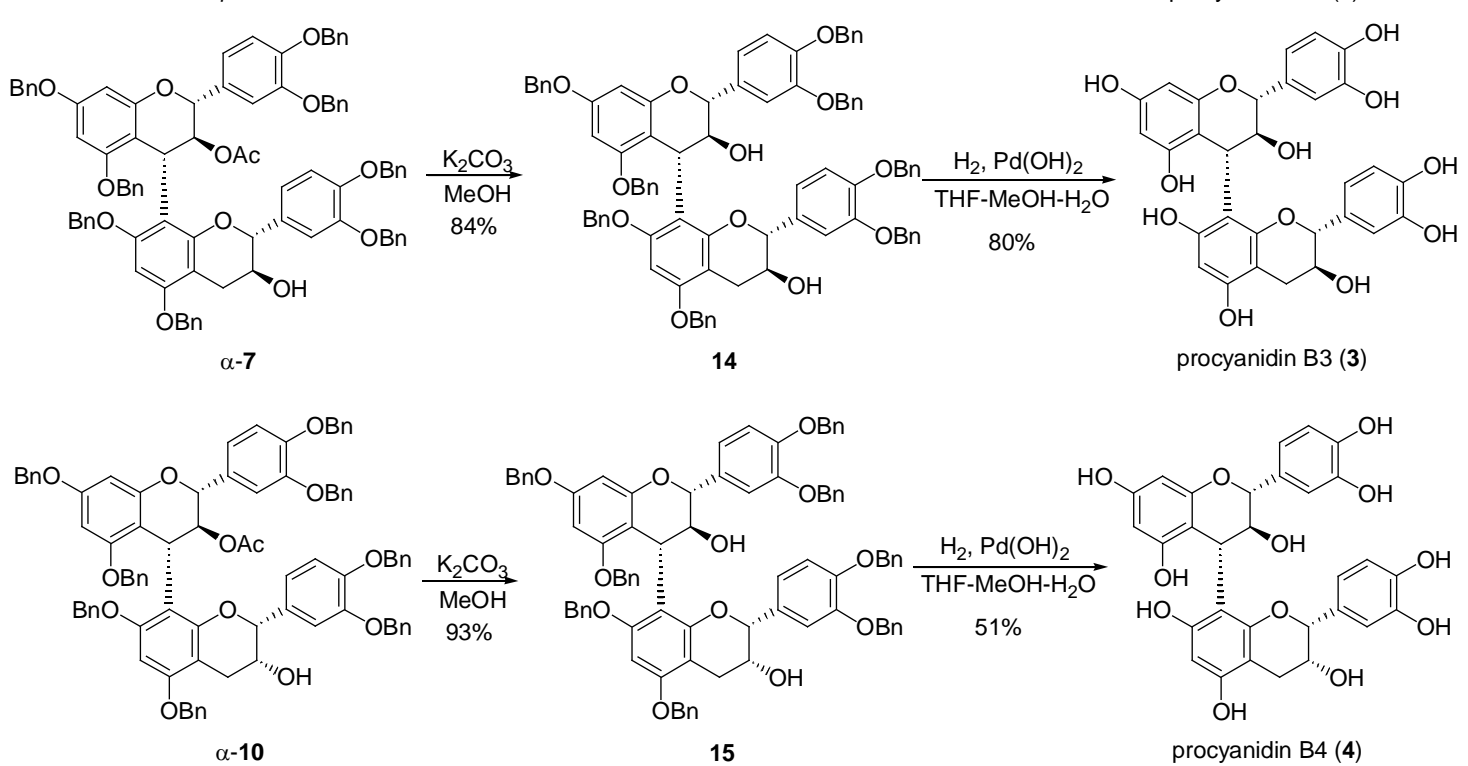

Scheme 2. Synthesis of procyanidin B1-B4 (1-4)

To characterize procyanidins in daily diet and evaluate their biological activities, it is necessary to obtain pure procyanidins. Synthetic procyanidins can be used as procyanidin standards. Recently, Hamauzu and co-workers reported the antiulceractive activity by apple phenolics in case of $\mathrm{HCl} /$ ethanol-induced 
ulcers. ${ }^{15}$ Thus, the synthesized authentic samples $1-4$ were used to clarify the structure of procyanidins from juice of apple. As shown in Figure 2 and 3, procyanidin B2 was identified as a major constituent of procyanidin dimer by HPLC analysis (Figure 2, 3). ${ }^{16}$

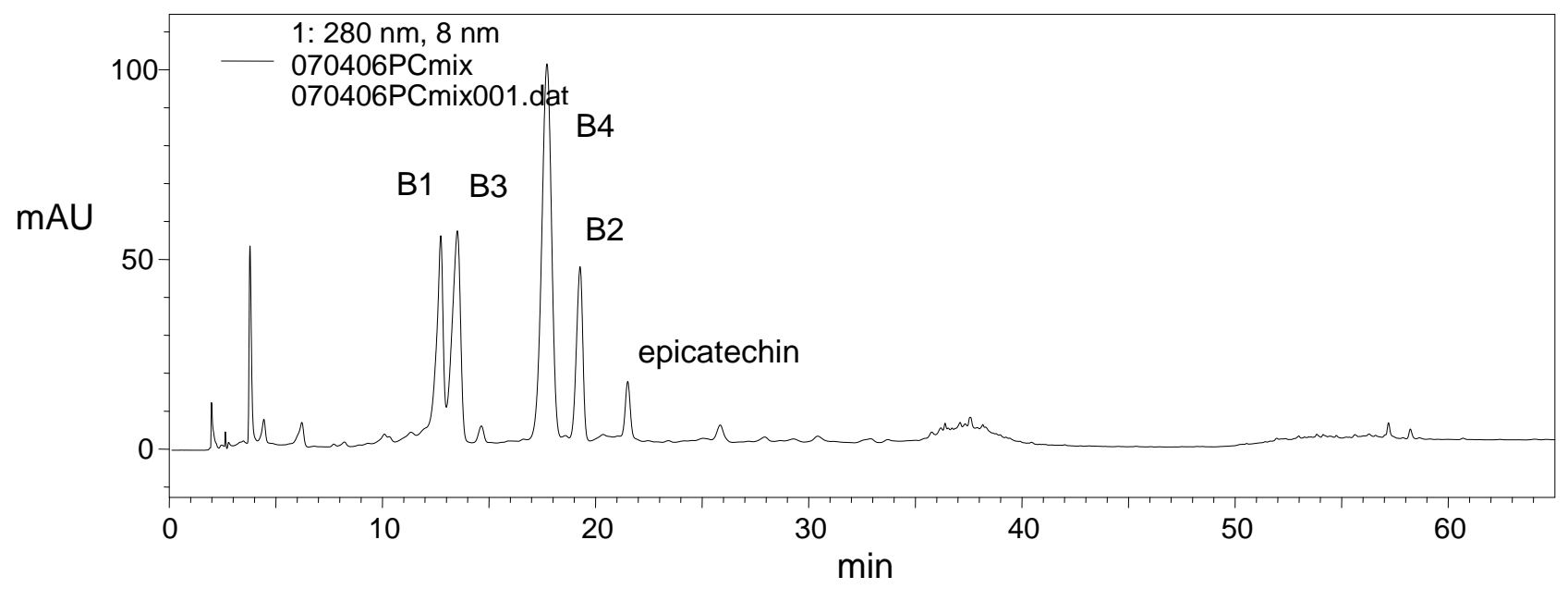

Figure 2. HPLC data of procyanidin B1-B4 (1-4).

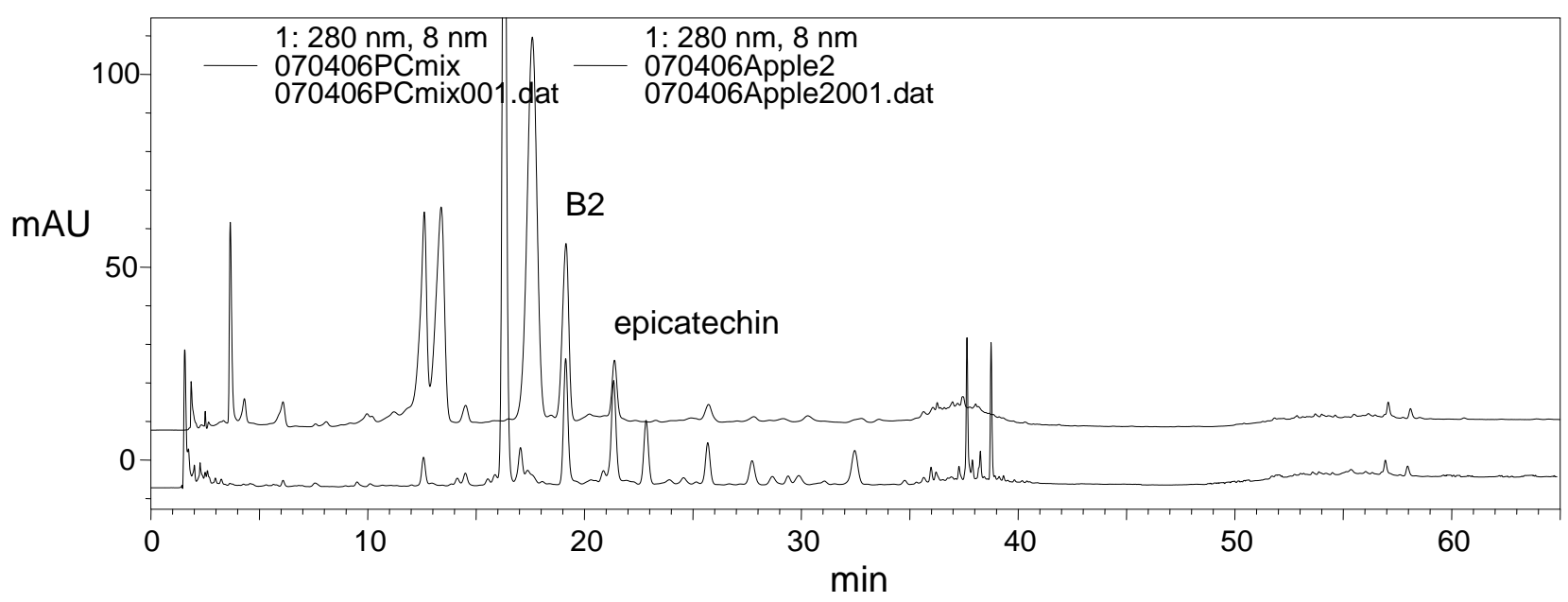

Figure 3. HPLC analysis of procyanidin dimers in the juice of apple.

\section{Conclusion}

In conclusion, we have synthesized procyanidin B1-B4 (1-4) based on a $\mathrm{Yb}(\mathrm{OTf})_{3}$ catalyzed equimolar condensation. The synthesis of various procyanidin oligomers according to the above-mentioned method and the structural identification of polyphenols from natural source are in progress. 


\section{EXPERIMENTAL}

General

All melting points were uncorrected. ${ }^{1} \mathrm{H}$ and ${ }^{13} \mathrm{C}$ NMR spectra were measured with a Bruker DRX 500 FT-NMR spectrometer in $\mathrm{CDCl}_{3}$ at 500 and $125 \mathrm{MHz}$, respectively. Chemical shifts were relative to tetramethylsilane as an internal standard. The coupling constants were given in $\mathrm{Hz}$. Mass spectra were obtained on JEOL JMS-700 mass spectrometer. IR spectra were recorded with JASCO FT-IR 480 Plus infrared spectrometer. Optical rotations were determined with a JASCO DIP-1000 polarimeter.

Representative procedure for $\mathrm{Yb}(\mathrm{OTf})_{3}$ catalyzed condensation; [4, 8']-2, 3-trans-3, 4-trans : 2', 3'-trans-Octa-O-benzyl-3-O-acetyl-bi-(+)-catechin ( $\boldsymbol{\alpha}-\mathbf{- 7})$. To a solution of nucleophile 5a (190 mg, $0.263 \mathrm{mmol})$ and electrophile 6a $(171 \mathrm{mg}, 0.263 \mathrm{mmol})$ in $\mathrm{CH}_{2} \mathrm{Cl}_{2}(10 \mathrm{~mL})$ under an argon atmosphere was added $\mathrm{Yb}(\mathrm{OTf})_{3}(163 \mathrm{mg}, 0.263 \mathrm{mmol})$. After the resulting mixture had been stirred for $2 \mathrm{~h}$, the reaction was quenched with water. The mixture was extracted with $\mathrm{Et}_{2} \mathrm{O}$, and the combined organic layer were washed with brine, dried over $\mathrm{MgSO}_{4}$, and concentrated. The crude product was purified with silica gel chromatography (hexane:AcOEt: $\left.\mathrm{CH}_{2} \mathrm{Cl}_{2}=4: 1: 2\right)$ to give diastereomeric mixture $\alpha-7$ and $\beta-7$ (226 mg, 64\%) as a colorless oil. ${ }^{1} \mathrm{H}$ NMR analysis of diacetate derivative showed more than 98:2 ratio of $\alpha-7$ and $\beta-7 .{ }^{4 b, 9}$ The selectivity was determined by ${ }^{1} \mathrm{H}$ NMR analysis of C-3 position of diacetate derivative of $\alpha-7$ (5.80 and $5.83 \mathrm{ppm})$ and $\beta-7$ (5.53 and $5.58 \mathrm{ppm})$ according to the reported procedure. ${ }^{4 \mathrm{~b}}$ Further purification with preparative TLC (hexane:AcOEt: $\mathrm{CH}_{2} \mathrm{Cl}_{2}=4: 1: 2$ ) gave $\alpha-7$. $[\alpha]_{\mathrm{D}}{ }^{24}-118$ (c 1.36, $\mathrm{CHCl}_{3}$ ). IR (film) $v_{\max } \mathrm{cm}^{-1}:$ 3523, 3087, 2909, 2870, 1731, 1607, 1511, 1498, 1454, 1428, 1373, 1306, 1264, 1231, 1139, 1113, 1063, 1027, 911, 849, 809, 735, 697, 606. ${ }^{1} \mathrm{H}$ NMR (500 MHz, $\mathrm{CDCl}_{3}, 1: 1$ mixture of rotational isomers): $\delta=7.49-7.12(40 \mathrm{H}, \mathrm{m}), 6.98-6.67(5.5 \mathrm{H}, \mathrm{m}), 6.43(0.5 \mathrm{H}, \mathrm{dd}, J=1.7,8.3$ Hz), 6.25 (0.5H, s), 6.23 (0.5H, d, $J=2.3 \mathrm{~Hz}), 6.20(0.5 \mathrm{H}, \mathrm{d}, J=2.3 \mathrm{~Hz}), 6.14(0.5 \mathrm{H}, \mathrm{d}, J=2.3 \mathrm{~Hz}), 6.11$ $(0.5 \mathrm{H}, \mathrm{d}, J=2.3 \mathrm{~Hz}), 5.99(0.5 \mathrm{H}, \mathrm{s}), 5.98(0.5 \mathrm{H}, \mathrm{t}, J=9.6 \mathrm{~Hz}), 5.86(0.5 \mathrm{H}, \mathrm{t}, J=9.6 \mathrm{~Hz}), 5.22-4.54$ (18.5H, m), 3.93 (0.5H, m), $3.58(0.5 \mathrm{H}, \mathrm{m}), 3.37$ (0.5H, d, $J=8.8 \mathrm{~Hz}), 3.04(0.5 \mathrm{H}, \mathrm{dd}, J=5.7,16.2 \mathrm{~Hz})$, $2.86(0.5 \mathrm{H}, \mathrm{dd}, J=5.0,16.5 \mathrm{~Hz}), 2.73$ (0.5H, dd, $J=7.0,16.5 \mathrm{~Hz}), 2.35$ (0.5H, dd, $J=9.6,16.1 \mathrm{~Hz}), 2.17$ (0.5H, m), 1.62 (1.5H, s), 1.54 (1.5H, s), 1.33 (0.5H, d, $J=2.3 \mathrm{~Hz}) .{ }^{13} \mathrm{C}$ NMR (125 MHz, $\mathrm{CDCl}_{3}, 1: 1$ mixture of rotational isomers): $\delta=158.20,158.10,157.79,156.77,156.72,156.54,155.89,155.82$, 155.54, 153.69, 152.53, 149.25, 149.04, 148.99, 148.94, 148.88, 148.84, 148.61, 137.95, 137.36, 137.31, 137.28, 137.26, 137.23, 137.07, 137.03, 136.97, 136.84, 136.59, 131.78, 131.59, 130.92, 128.51, 128.48, 128.44, 128.40, 128.36, 128.33, 128.31, 128.28, 128.11, 128.04, 127.96, 127.82, 127.76, 127.69, 127.60, 127.52, 127.47, 127.46, 127.43, 127.33, 127.28, 127.26, 127.19, 127.17, 127.09, 126.97, 126.85, 121.27, 120.92, 120.30, 120.05, 114.96, 114.79, 114.61, 114.45, 114.19, 114.07, 113.67, 110.74, 110.27, 108.29, 107.94, 102.34, 102.25, 94.91, 94.85, 94.42, 94.33, 91.24, 91.19, 80.64, 80.19, 80.10, 79.86, 71.57, 71.50, 
71.35, 71.27, 71.15, 70.39, 70.26, 70.01, 69.98, 69.76, 68.84, 67.75, 35.40, 35.16, 28.62, 26.49, 20.65, 20.40. FAB-HRMS: Calcd for $\mathrm{C}_{88} \mathrm{H}_{77} \mathrm{O}_{13}[\mathrm{M}+\mathrm{H}]^{+}, 1341.5364$; found, 1341.5381 .

\section{[4, 8']-2, 3-cis-3, 4-trans : 2', 3'-trans-Octa-O-benzyl-3-O-acetyl-(-)-epicatechin-(+)-catechin ( $\beta$-8).}

In the same manner as prepared $\alpha-7$, compounds $5 \mathrm{a}(72 \mathrm{mg}, 0.11 \mathrm{mmol})$ and $6 \mathrm{~b}(80 \mathrm{mg}, 0.11 \mathrm{mmol})$ gave $\alpha-8$ and $\beta-8$ (92 mg, 62\%, $\alpha-8: \beta-8=9: 91$ ) in 62\% yield. Further purification with preparative TLC (hexane:AcOEt: $\left.\mathrm{CH}_{2} \mathrm{Cl}_{2}=4: 1: 2\right)$ gave pure $\beta-8 .{ }^{4 \mathrm{~d}}[\alpha]_{\mathrm{D}}{ }^{18}+66.0\left(c 1.47, \mathrm{CHCl}_{3}\right.$ ). IR (film) $v_{\max } \mathrm{cm}^{-1}: 3568$, 3062, 3031, 2927, 2870, 1740, 1594, 1513, 1498, 1454, 1425, 1376, 1331, 1265, 1216, 1119, 1073, 1028, 910, 851, 809, 737, 697, 625. ${ }^{1} \mathrm{H} \mathrm{NMR}\left(500 \mathrm{MHz} \mathrm{CDCl}_{3}, 0.71: 0.29\right.$ mixture of rotational isomers): $\delta=7.43-6.75(43.29 H, \mathrm{~m}), 6.51-6.49(0.71 \mathrm{H}, \mathrm{m}), 6.30(0.71 \mathrm{H}, \mathrm{s}), 6.22(0.29 \mathrm{H}, \mathrm{s}), 6.18(0.29 \mathrm{H}, \mathrm{s}), 6.12$ (0.29H, s), $6.03(0.71 \mathrm{H}, \mathrm{d}, J=2.0 \mathrm{~Hz}), 5.54(0.71 \mathrm{H}, \mathrm{d}, J=2.0 \mathrm{~Hz}), 5.52(0.71 \mathrm{H}, \mathrm{s}), 5.45(0.71 \mathrm{H}, \mathrm{s}), 5.37$ (0.29H, s), $5.26(0.29 \mathrm{H}, \mathrm{s}), 5.08-4.51(20.58 \mathrm{H}, \mathrm{m}), 3.78(0.71 \mathrm{H}, \mathrm{dd}, J=9.1,16.0 \mathrm{~Hz}), 3.65(0.71 \mathrm{H}, \mathrm{d}, J=$ $9.1 \mathrm{~Hz}), 3.24(0.71 \mathrm{H}, \mathrm{dd}, J=6.5,16.8 \mathrm{~Hz}), 2.68$ (0.29H, dd, $J=9.6,16.5 \mathrm{~Hz}), 1.69$ (2.13H, s), 1.63 $(0.29 \mathrm{H}, \mathrm{br}, \mathrm{OH}), 1.53(0.87 \mathrm{H}, \mathrm{s}), 1.27(0.71 \mathrm{H}, \mathrm{d}, J=12.4 \mathrm{~Hz}, \mathrm{OH}) .{ }^{13} \mathrm{C} \mathrm{NMR}\left(125 \mathrm{MHz}, \mathrm{CDCl}_{3}\right.$, 0.71:0.29 mixture of rotational isomers): $\delta=169.06,158.07,156.46,156.12,156.02,155.56,154.37$, 149.33, 149.04, 148.85, 148.69, 137.39, 137.32, 137.22, 137.07, 132.18, 130.44, 128.93, 128.61, 128.58, 128.44, 128.40, 128.37, 128.31, 128.17, 128.07, 127.93, 127.72, 127.67, 127.56, 127.50, 127.42, 127.35, 127.31, 127.22, 127.19, 127.17, 127.09, 127.03, 120.63, 119.81, 114.76, 114.66, 114.19, 113.67, 112.10, 110.66, 104.47, 104.13, 93.62, 92.85, 91.51, 81.78, 79.18, 74.53, 72.09, 71.49, 71.35, 71.09, 70.86, 70.64, 70.35, 69.98, 69.63, 69.29, 68.67, 33.31, 29.67, 29.09, 20.73. HRFABMS calcd for $\mathrm{C}_{88} \mathrm{H}_{76} \mathrm{O}_{13} \mathrm{Na}$ $[\mathrm{M}+\mathrm{Na}]^{+} ; 1363.5183$; found 1363.5138 .

[4, 8']-2, 3-cis-3, 4-trans : 2', 3'-cis-Octa-O-benzyl-3-O-acetyl-bi-(-)-epicatechin ( $\beta$-9). In the same manner as prepared $\alpha-7$, compounds $5 \mathbf{b}$ (36 mg, $0.05 \mathrm{mmol}$ ) and $\mathbf{6 b}$ (33 $\mathrm{mg}, 0.050 \mathrm{mmol}$ ) gave $\alpha-\mathbf{9}$ and $\beta$-9 (51 mg, 76\%, $\alpha-\mathbf{9}: \beta-9=12: 88$ ). Further purification with preparative TLC (hexane:AcOEt: $\mathrm{CH}_{2} \mathrm{Cl}_{2}=$ 4:1:2) gave pure $\beta-9 .{ }^{4 \mathrm{~d}}[\alpha]_{\mathrm{D}}{ }^{18}+39.1$ (c 2.59, $\mathrm{CHCl}_{3}$ ). IR (film) $v_{\max } \mathrm{cm}^{-1}: 3528,3092,2914,2875,1736$, 1612, 1516, 1503, 1459, 1433, 1378, 1311, 1269, 1236, 1143, 1118, 1068, 1032, 916, 854, 814, 740, 702, 611. ${ }^{1} \mathrm{H}$ NMR (500 MHz, $\mathrm{CDCl}_{3}, 0.82: 0.18$ mixture of rotational isomers): $\delta=7.43-6.80$ (43H, m), 6.44 (0.82H, d, $J=8.1 \mathrm{~Hz}), 6.30(0.82 \mathrm{H}, \mathrm{s}), 6.27$ (0.18H, d, $J=1.92 \mathrm{~Hz}), 6.23(0.18 \mathrm{H}, \mathrm{s}), 6.19(0.18 \mathrm{H}, \mathrm{s}), 6.12$ (0.18H, d, $J=1.0 \mathrm{~Hz}), 6.01(0.82 \mathrm{H}, \mathrm{br}), 5.68(0.82 \mathrm{H}, \mathrm{br}), 5.63(0.82 \mathrm{H}, \mathrm{br}), 5.51(0.82 \mathrm{H}, \mathrm{br}), 5.37$ (0.18H, br), $5.33(0.18 \mathrm{H}, \mathrm{br}), 5.17-4.75(20.18 \mathrm{H}, \mathrm{m}), 4.64(0.82 \mathrm{H}, \mathrm{d}, J=10.9 \mathrm{~Hz}), 4.46(0.82 \mathrm{H}, \mathrm{d}, J=11.3 \mathrm{~Hz})$, 4.21-4.04 (0.36H, m), 3.88 (0.82H, br), 3.02-2.88 (2H, m), 1.70 (2.46H, s), 1.65 (0.18H, d, J = 4.55 Hz, $\mathrm{OH}), 1.49$ (0.82H, br, OH), $1.35(0.54 \mathrm{H}, \mathrm{s}) .{ }^{13} \mathrm{C} \mathrm{NMR}\left(125 \mathrm{MHz}, \mathrm{CDCl}_{3}, 0.82: 0.18\right.$ mixture of rotational isomers): $\delta=158.33,158.05,156.67,156.54,156.02,155.45,155.28,154.43,149.05,148.92,148.71$, 
148.62, 148.29, 137.41, 137.37, 137.33, 137.30, 137.19, 137.10, 136.96, 132.16, 131.27, 128.60, 128.57, 128.51, 128.47, 128.45, 128.38, 128.14, 128.01, 127.92, 127.83, 127.79, 127.72, 127.67, 127.64, 127.52, 127.50, 127.43, 127.35, 127.32, 127.26, 127.18, 127.14, 126.98, 126.72, 125.85, 119.99, 119.84, 119.53, 118.91, 115.19, 114.82, 114.60, 113.83, 113.66, 112.58, 110.60, 104.69, 102.16, 94.77, 94.04, 93.35, 92.98, 92.75, 91.55, 79.07, 78.37, 74.94, 74.75, 72.18, 71.54, 71.43, 71.36, 71.23, 71.17, 70.59, 70.16, 69.99, 69.81, 69.58, 69.22, 66.56, 66.34, 65.81, 33.32, 28.73, 20.74, 20.29. HRFABMS calcd for $\mathrm{C}_{88} \mathrm{H}_{77} \mathrm{O}_{13}[\mathrm{M}+\mathrm{H}]^{+}$; 1341.5364 ; found 1341.5391 .

\section{[4, 8']-2, 3-trans-3, 4-trans : 2', 3'-cis-Octa-O-benzyl-3-O-acetyl-(+)-catechin-(-)-epicatechin $(\alpha-10)$.}

In the same manner as prepared $\alpha-7$, compounds $5 \mathbf{b}(42 \mathrm{mg}, 0.064 \mathrm{mmol})$ and $6 \mathbf{a}(46 \mathrm{mg}, 0.064 \mathrm{mmol})$ gave $\alpha-\mathbf{1 0}$ and $\beta-\mathbf{1 0}$ (56 mg, 59\%, $\alpha-\mathbf{1 0}: \beta-\mathbf{1 0}=98: 2)$. Further purification with preparative TLC (hexane:AcOEt: $\mathrm{CH}_{2} \mathrm{Cl}_{2}=4: 1: 2$ ) gave pure $\beta-\mathbf{1 0}^{4 \mathrm{~d}}[\alpha]_{\mathrm{D}}{ }^{18}-78.0$ (c $0.52, \mathrm{CHCl}_{3}$ ). IR (film) $v_{\max } \mathrm{cm}^{-1}$ : 3568, 3062, 3031, 2869, 1741, 1607, 1511, 1454, 1429, 1373, 1265, 1227, 1139, 1113, 1028, 911, 849, 736, 697, 619. ${ }^{1} \mathrm{H}$ NMR (500 $\mathrm{MHz}, \mathrm{CDCl}_{3}, 0.67: 0.33$ mixture of rotational isomers): $\delta=7.44-6.77$ (44.65H, m), $6.60(0.33 \mathrm{H}, \mathrm{dd}, J=1.5,8.3 \mathrm{~Hz}), 6.23-6.21(2.01 \mathrm{H}, \mathrm{m}), 6.13(0.67 \mathrm{H}, \mathrm{d}, J=2.3 \mathrm{~Hz})$, 5.92-5.90 (0.67H, m), 5.80-5.76 (0.67H, m), 5.22-4.47 (29.37H, m), 4.08 (0.33H, br), 3.91 $(0.67 \mathrm{H}, \mathrm{br})$, 3.59 (0.67H, br), 2.99-2.91 (0.67H, m), 2.80 (0.67H, d, $J=16.8 \mathrm{~Hz}), 2.56(0.67 \mathrm{H}, \mathrm{dd}, J=4.5,17.1 \mathrm{~Hz})$, $1.57(2.01 \mathrm{H}, \mathrm{s}), 1.50(0.99 \mathrm{H}, \mathrm{s}), 1.44(0.33 \mathrm{H}, \mathrm{d}, J=6.3 \mathrm{~Hz}), 1.36(0.67 \mathrm{H}, \mathrm{d}, J=5.8 \mathrm{~Hz}) .{ }^{13} \mathrm{C}$ NMR $(125$ $\mathrm{MHz}, \mathrm{CDCl}_{3}, 0.67: 0.33$ mixture of rotational isomers): $\delta=169.21,158.30,158.14,157.82,156.66$, 156.31, 156.22, 156.05, 155.78, 153.21, 149.06, 149.02, 148.94, 148.81, 148.47, 137.43, 137.39, 137.36, 137.31, 137.29, 137.25, 137.19, 137.13, 137.08, 136.94, 136.56, 132.45, 131.03, 131.93, 128.67, 128.55, 128.51, 128.49, 128.47, 128.38, 128.33, 128.11, 128.07, 127.90, 127.88, 127.84, 127.77, 127.68, 127.62, 127.58, 127.52, 127.47, 127.44, 127.34, 127.28, 127.25, 127.11, 127.02, 126.88, 121.28, 120.92, 119.75, 119.03, 115.11, 114.83, 114.78, 114.01, 113.52, 111.10, 110.59, 108.48, 108.05, 102.17, 100.48, 94.92, 94.34, 91.61, 91.39, 80.19, 79.94, 78.24, 73.26, 71.75, 71.46, 71.38, 71.28, 71.22, 71.15, 70.87, 70.66, 70.36, 70.23, 70.19, 70.01, 69.95, 69.63, 66.79, 65.86, 35.30, 35.12, 28.59, 20.64, 20.39. HRFABMS calcd for $\mathrm{C}_{88} \mathrm{H}_{76} \mathrm{O}_{13} \mathrm{Na}[\mathrm{M}+\mathrm{Na}]^{+}$; 1363.5183 ; found 1363.5146 .

[4, 8']-2, 3-cis-3, 4-trans : 2', 3'-trans-Octa-O-benzyl-(-)-epicatechin-(+)-catechin (12). To a solution of $\beta-8$ (78 mg, $0.057 \mathrm{mmol})$ in $\mathrm{MeOH}(10 \mathrm{~mL})$ was added $\mathrm{K}_{2} \mathrm{CO}_{3}(156 \mathrm{mg}, 1.10 \mathrm{mmol})$. After being stirred for $12 \mathrm{~h}$ at $60{ }^{\circ} \mathrm{C}$, the mixture was diluted with $\mathrm{H}_{2} \mathrm{O}$ and extracted with diethyl ether. The organic layer was washed with water, brine, and dried with $\mathrm{MgSO}_{4}$. The solvent was evaporated and the residue was purified with preparative TLC (hexane:AcOEt: $\left.\mathrm{CH}_{2} \mathrm{Cl}_{2}=4: 1: 2\right)$ to afford 12 (60 mg, 82\%) as an

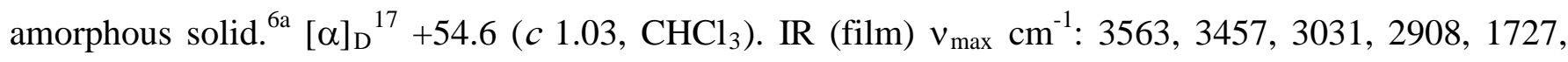


1594, 1513, 1497, 1454, 1423, 1379, 1265, 1214, 1118, 1072, 1027, 910, 851, 808, 736, 696, 625. ${ }^{1} \mathrm{H}$ NMR (500 MHz, $\mathrm{CDCl}_{3}, 0.71: 0.29$ mixture of rotational isomers): $\delta=7.49-7.10$ (36.29H, m), 7.06-6.87 $(6 \mathrm{H}, \mathrm{m}), 6.79-6.78(1 \mathrm{H}, \mathrm{m}), 6.75-6.72(1 \mathrm{H}, \mathrm{m}), 6.49(0.71 \mathrm{H}, \mathrm{dd}, J=1.7,8.1 \mathrm{~Hz}), 6.34(0.71 \mathrm{H}, \mathrm{s}), 6.21$ $(0.29 \mathrm{H}, \mathrm{d}, J=2.2 \mathrm{~Hz}), 6.17(0.29 \mathrm{H}, \mathrm{s}), 6.05(0.29 \mathrm{H}, \mathrm{d}, J=2.2 \mathrm{~Hz}), 6.02(0.71 \mathrm{H}, \mathrm{d}, J=2.2 \mathrm{~Hz}), 5.54$ $(0.71 \mathrm{H}, \mathrm{d}, J=2.1 \mathrm{~Hz}), 5.40(0.71 \mathrm{H}, \mathrm{s}), 5.31(0.29 \mathrm{H}, \mathrm{s}), 5.12-4.82(16 \mathrm{H}, \mathrm{m}), 4.67$ (0.29H, br), 4.64-4.60 $(1 \mathrm{H}, \mathrm{m}), 4.51(0.71 \mathrm{H}, \mathrm{d}, J=11.3 \mathrm{~Hz}), 4.40(0.29 \mathrm{H}, \mathrm{d}, J=12.0 \mathrm{~Hz}), 4.02(0.71 \mathrm{H}, \mathrm{d}, J=4.8 \mathrm{~Hz}), 3.87$ $(0.29 \mathrm{H}, \mathrm{d}, J=4.2 \mathrm{~Hz}), 3.76-3.71(0.71 \mathrm{H}, \mathrm{m}), 3.65-3.62(1 \mathrm{H}, \mathrm{m}), 3.24(0.71 \mathrm{H}, \mathrm{dd}, J=6.5,16.8 \mathrm{~Hz}), 3.17$ (0.29H, dd, $J=5.6,16.4 \mathrm{~Hz}), 2.68(0.29 \mathrm{H}, \mathrm{dd}, J=9.4,16.3 \mathrm{~Hz}), 2.58(0.71 \mathrm{H}, \mathrm{dd}, J=9.7,16.7 \mathrm{~Hz}), 1.76$ $(0.71 \mathrm{H}, \mathrm{d}, J=6.01 \mathrm{~Hz}), 1.61(0.29 \mathrm{H}, \mathrm{br}), 1.43$ (0.71H, br), 1.25 (0.29H, br). ${ }^{13} \mathrm{C} \mathrm{NMR}\left(125 \mathrm{MHz}, \mathrm{CDCl}_{3}\right.$, $0.71: 0.29$ mixture of rotational isomers): $\delta=158.28,158.08,157.74,156.97,156.85,155.90,155.82$, 155.60, 155.15, 154.42, 152.91, 149.12, 148.96, 148.78, 148.72, 148.63, 148.47, 137.34, 137.29, 137.25, 137.22, 137.20, 137.15, 137.06, 137.01, 136.97, 136.90, 132.61, 132.50, 130.97, 130.27, 128.61, 128.56, 128.55, 128.53, 128.45, 128.42, 128.40, 128.36, 128.19, 128.17, 128.10, 128.05, 127.93, 127.73, 127.68, 127.57, 127.48, 127.43, 127.35, 127.31, 127.21, 127.16, 127.14, 127.11, 127.08, 126.96, 126.89, 126.75, 120.52, 120.16, 119.95, 119.62, 114.95, 114.51, 113.98, 113.88, 113.54, 112.92, 112.07, 111.19, 104.57, 104.26, 104.19, 102.69, 94.34, 93.46, 93.18. 93.00, 92.60, 91.47, 81.58, 81.39, 75.56, 75.38, 72.28, 71.74, 71.44, 71.40, 71.32, 71.23, 71.15, 71.07, 70.76, 70.55, 70.24, 69.89, 69.57, 69.43, 69.11, 68.62, 68.31, 35.78, 35.73, 29.06, 27.75 .

[4, 8']-2, 3-cis-3, 4-trans : 2', 3'-cis-Octa-O-benzyl-bi-(-)-epicatechin (13). In the same manner as prepared 12, compound $\beta-9$ (48 mg, $0.034 \mathrm{mmol}$ ) gave 13 (40 mg, 90\%). ${ }^{4 \mathrm{~d}}[\alpha]_{\mathrm{D}}{ }^{18}+34.9\left(\mathrm{c} 2.18, \mathrm{CHCl}_{3}\right)$. IR (film) $v_{\max } \mathrm{cm}^{-1}:$ 3572, 3067, 3036, 2933, 2874, 1737, 1611, 1515, 1498, 1454, 1431, 1382, 1269, 1220, 1182, 1112, 1067, 1031, 915, 851, 809, 741, 697, 624. ${ }^{1} \mathrm{H}$ NMR (500 MHz, $\mathrm{CDCl}_{3}, 0.6: 0.4$ mixture of rotational isomers): $\delta=7.43-6.83(42.8 \mathrm{H}, \mathrm{m}), 6.80(0.6 \mathrm{H}, \mathrm{d}, J=8.2 \mathrm{~Hz}), 6.44(0.6 \mathrm{H}, \mathrm{s}), 6.34(0.6 \mathrm{H}, \mathrm{s})$, $6.22(0.4 \mathrm{H}, \mathrm{d}, J=2.3 \mathrm{~Hz}), 6.17(0.4 \mathrm{H}, \mathrm{s}), 6.07(0.4 \mathrm{H}, \mathrm{d}, J=2.2 \mathrm{~Hz}), 6.00(0.6 \mathrm{H}, \mathrm{d}, J=2.2 \mathrm{~Hz}), 5.70$ (0.6H, d, $J=2.2 \mathrm{~Hz}), 5.52(0.6 \mathrm{H}, \mathrm{s}), 5.33(0.4 \mathrm{H}, \mathrm{s}), 5.20-4.86$ (15.8H, m), $4.79(1.2 \mathrm{H}, \mathrm{s}), 4.62$ (1H, d, $J=$ $11.2 \mathrm{~Hz}), 4.47$ (0.6H, d, $J=11.3), 4.38(0.4 \mathrm{H}, \mathrm{d}, J=12.0 \mathrm{~Hz}), 4.32(0.4 \mathrm{H}, \mathrm{br}), 4.06(1 \mathrm{H}, \mathrm{br}), 3.96(0.4 \mathrm{H}$, br), $3.86(0.6 \mathrm{H}, \mathrm{d}, J=3.7 \mathrm{~Hz}), 3.06-2.87(2 \mathrm{H}, \mathrm{m}), 1.77$ (0.6H, br), 1.69 (0.4H, d, $J=5.3 \mathrm{~Hz}), 1.50$ (0.6H, br), $1.44(0.4 \mathrm{H}, \mathrm{br}) .{ }^{13} \mathrm{C} \mathrm{NMR}\left(125 \mathrm{MHz}, \mathrm{CDCl}_{3}, 0.6: 0.4\right.$ mixture of rotational isomers): $\delta=158.08$, 158.05, 157.87, 157.10, 157.04, 156.56, 156.48, 155.91, 155.86, 155.50, 155.04, 154.41, 149.20, 149.13, 148.73, 148.63, 148.50, 148.36, 148.26, 148.14, 137.39, 137.35, 137.32, 137.30, 137.26, 137.24, 137.19, 137.14, 136.98, 136.94, 132.67, 132.59, 131.16, 131.10, 128.58, 128.56, 128.54, 128.49, 128.47, 128.42, 128.38, 128.34, 128.30, 128.15, 128.12, 127.98, 127.96, 127.88, 127.83, 127.69, 127.66, 127.56, 127.50, 127.48, 127.44, 127.34, 127.32, 127.23, 127.17, 127.14, 127.12, 127.00, 126.97, 126.95, 126.90, 126.75, 
126.59, 119.85, 119.76, 118.84, 118.76, 118.63, 115.15, 114.99, 114.86, 114.53, 114.32, 113.49, 112.61, 112.43, 111.65, 111.28, 111.14, 104.52, 104.42, 102.35, 102.26, 94.48, 93.93, 93.31, 93.18, 93.12, 91.50, 78.87, 78.81, 78.05, 75.64, 75.61, 72.39, 72.12, 71.56, 71.43, 71.32, 71.20, 70.93, 70.80, 70.55, 70.46, 69.98, 69.90, 69.84, 69.75, 69.52, 69.47, 69.11, 69.03, 66.50, 65.16, 35.83, 35.74, 28.64.

[4, 8']-2, 3-trans-3, 4-trans : 2', 3'-trans-Octa-O-benzyl-bi-(+)-catechin (14). In the same manner as prepared 12, compound $\alpha-7$ (214mg, $0.155 \mathrm{mmol}$ ) gave 14 (169 mg, 84\%). ${ }^{9}[\alpha]_{\mathrm{D}}{ }^{24}-127.5$ (c 1.00, $\mathrm{CHCl}_{3}$ ). IR (film) $v_{\max } \mathrm{cm}^{-1}:$ 3567, 3062, 3031, 2928, 2869, 1732, 1606, 1510, 1498, 1454, 1426, 1377 , 1264, 1215, 1177, 1112, 1062, 1026, 910, 850, 809, 736, 697, 623. ${ }^{1} \mathrm{H}$ NMR (500 MHz, CDCl 3 , 0.67:0.33 mixture of rotational isomers): $\delta=7.49-7.15(40 \mathrm{H}, \mathrm{m}), 7.04-6.47(6 \mathrm{H}, \mathrm{m}), 6.31(0.67 \mathrm{H}, \mathrm{s}), 6.23(0.33 \mathrm{H}, \mathrm{d}$, $J=2.3 \mathrm{~Hz}), 6.20(0.67 \mathrm{H}, \mathrm{d}, J=2.3 \mathrm{~Hz}), 6.13(1 \mathrm{H}, \mathrm{d}, J=2.4 \mathrm{~Hz}), 6.04(0.33 \mathrm{H}, \mathrm{d}, J=2.3 \mathrm{~Hz}), 5.20-4.48$ $(18 \mathrm{H}, \mathrm{m}), 4.32(0.67 \mathrm{H}, \mathrm{m}), 4.20(0.33 \mathrm{H}, \mathrm{m}), 3.75-3.70(1 \mathrm{H}, \mathrm{m}), 3.67(1 \mathrm{H}, \mathrm{d}, J=8.5 \mathrm{~Hz}), 3.20(0.33 \mathrm{H}, \mathrm{dd}$, $J=5.9,16.4 \mathrm{~Hz}), 3.08(0.67 \mathrm{H}, \mathrm{dd}, J=5.6,16.2 \mathrm{~Hz}), 2.68(0.33 \mathrm{H}, \mathrm{dd}, J=9.4,16.4 \mathrm{~Hz}), 2.42(0.67 \mathrm{H}, \mathrm{dd}$,

$J=9.1,16.2 \mathrm{~Hz}) .{ }^{13} \mathrm{C} \mathrm{NMR}\left(125 \mathrm{MHz}, \mathrm{CDCl}_{3}, 0.67: 0.33\right.$ mixture of rotational isomers): $\delta=158.00$, 157.73, 156.98, 156.84, 155.58, 155.53, 153.86, 152.85, 149.28, 149.16, 149.04, 148.66, 137.69, 137.32, 137.30, 137.18, 137.14, 137.04, 136.70, 131.92, 131.74, 128.57, 128.53, 128.51, 128.47, 128.41, 128.38, 128.35, 128.33, 128.15, 128.06, 127.87, 127.84, 127.82, 127.78, 127.75, 127.71, 127.67, 127.61, 127.56, 127.51, 127.46, 127.44, 127.27, 127.23, 127.22, 127.11, 127.08, 127.05, 121.28, 120.82, 120.69, 120.11, 115.20, 115.02, 114.98, 114.70, 114.24, 113.90, 113.71, 112.16, 108.70, 108.53, 102.52, 94.97, 94.18, 91.89, 91.59, 82.06, 81.76, 81.27, 80.66, 73.42, 73.30, 71.38, 71.24, 71.21, 71.16, 71.04, 70.37, 70.13, 70.02, 69.98, 69.91, 68.49, 68.41.

[4, 8']-2, 3-trans-3, 4-trans : 2', 3'-cis-Octa-O-benzyl-(+)-catechin-(-)-epicatechin (15). In the same manner as prepared 12, compound $\alpha-10$ (53 mg, $0.038 \mathrm{mmol})$ gave $15(46 \mathrm{mg}, 93 \%){ }^{6 \mathrm{a}}[\alpha]_{\mathrm{D}}{ }^{19}-96.9(c$ 1.70, $\mathrm{CHCl}_{3}$ ). IR (film) $v_{\max } \mathrm{cm}^{-1}:$ 3567, 3063, 3032, 2912, 2870, 1741, 1607, 1512, 1454, 1427, 1379, 1266, 1218, 1107, 1058, 1027, 910, 849, 811, 735, 697, 624. ${ }^{1} \mathrm{H}$ NMR (500 MHz, $\mathrm{CDCl}_{3}, 0.71: 0.29$ mixture of rotational isomers) $: \delta=7.45-6.78(48 \mathrm{H}, \mathrm{m}), 6.47(0.29 \mathrm{H}, \mathrm{d}, J=8.3 \mathrm{~Hz}), 6.22-6.21(1 \mathrm{H}, \mathrm{m})$, $6.19(0.71 \mathrm{H}, \mathrm{d}, J=2.1 \mathrm{~Hz}), 6.12(0.71 \mathrm{H}, \mathrm{d}, J=2.1 \mathrm{~Hz}), 5.99(0.29 \mathrm{H}, \mathrm{s}), 5.18(0.71 \mathrm{H}, \mathrm{d}, J=12.1 \mathrm{~Hz})$, $5.13(2 \mathrm{H}, \mathrm{d}, J=5.6 \mathrm{~Hz}), 5.10(1.71 \mathrm{H}, \mathrm{s}), 5.08(1 \mathrm{H}, \mathrm{s}), 5.05-4.47(12.87 \mathrm{H}, \mathrm{m}), 4.27(1 \mathrm{H}, \mathrm{dd}, J=8.8,18.0$ Hz), $4.09(0.29 \mathrm{H}, \mathrm{br}), 3.88(0.71 \mathrm{H}, \mathrm{br}), 3.79(0.71 \mathrm{H}, \mathrm{s}), 3.01(0.29 \mathrm{H}, \mathrm{d}, J=17.1 \mathrm{~Hz}), 2.92(0.29 \mathrm{H}, \mathrm{d}, J=$ $4.6 \mathrm{~Hz}), 2.87(0.71 \mathrm{H}, \mathrm{d}, J=17.3 \mathrm{~Hz}), 2.59(0.71 \mathrm{H}, \mathrm{dd}, J=4.4,17.1 \mathrm{~Hz}), 1.69-1.39(2 \mathrm{H}, \mathrm{m}) .{ }^{13} \mathrm{C}$ NMR (125 MHz, $\mathrm{CDCl}_{3}, 0.71: 0.29$ mixture of rotational isomers) $: \delta=158.21,158.09,158.00,157.81,156.95$, 156.84, 156.56, 156.13, 156.04, 155.49, 153.66, 152.89, 149.25, 149.16, 149.13, 149.04, 148.99, 148.78, 148.33, 137.66, 137.44, 137.41, 137.34, 137.30, 137.27, 137.24, 137.22, 137.19, 137.16, 137.13, 137.09, 
136.99, 136.84, 136.70, 132.17, 131.93, 131.87, 131.09, 128.56, 128.55, 128.52, 128.48, 128.44, 128.42, 128.40, 128.37, 128.34, 128.13, 128.09, 128.04, 127.86, 127.85, 127.74, 127.71, 127.63, 127.59, 127.55, 127.52, 127.44, 127.25, 127.23, 127.21, 127.13, 127.11, 127.08, 121.29, 120.93, 119.95, 118.79, 115.31, 115.05, 114.98, 114.61, 114.00, 113.78, 113.17, 112.08, 111.74, 108.84, 108.28, 102.25, 100.88, 95.41, 94.96, 94.44, 94.16, 92.17, 91.57, 82.09, 81.86, 77.45, 77.25, 73.19, 72.52, 71.86, 71.50, 71.35, 71.24, 71.20, 71.13, 71.01, 70.37, 70.25, 70.15, 70.04, 69.99, 69.95, 69.73, 66.29, 65.96, 37.46, 37.06, 29.67, 28.85, 28.29, 22.66.

Procyanidin B1 (1). Compound 12 (60 mg, $0.046 \mathrm{mmol})$ and $\mathrm{Pd}(\mathrm{OH})_{2}$ on carbon (20wt\%, $\left.12 \mathrm{mg}\right)$ in THF-MeOH- $\mathrm{H}_{2} \mathrm{O}$ (20:1:1, $10 \mathrm{~mL}$ ) was stirred for $48 \mathrm{~h}$ under the $\mathrm{H}_{2}$ atmosphere. After the reaction had been completed the mixture was filtered and the solvent was evaporated. The residue was purified with ODS cartridge column chromatography ( $\mathrm{MeOH}: \mathrm{H}_{2} \mathrm{O}=3: 7$ ) afforded 1 (20 mg, 76\%) as a colorless solid. Mp 184.5-185.0 ${ }^{\circ} \mathrm{C}$ (decomp.); $[\alpha]_{\mathrm{D}}{ }^{20}+43.3$ (c 0.27, EtOH); ${ }^{1} \mathrm{H}$ NMR (500 MHz, $\left.\mathrm{CD}_{3} \mathrm{OD}\right): \delta=2.57-2.63$ (1H, m), 2.77 (1H, m), 3.94 (1H, m), 3.98-4.12 (1H, m), 4.56-4.60 (1H, m), $4.80(1 \mathrm{H}, \mathrm{m}), 5.10(1 \mathrm{H}, \mathrm{m})$, 5.86-5.93 (3H, m), 6.69-6.84 (5H, m), 6.90 (1H, m); ${ }^{13} \mathrm{C}-\mathrm{NMR}\left(125 \mathrm{MHz}, \mathrm{CD}_{3} \mathrm{OD}\right) \delta=27.73,30.20$, 37.22, 68.64, 73.19, 77.13, 79.02, 79.28, 79.54, 82.49, 95.95, 96.40, 97.05, 101.32, 115.13, 115.40, 115.96, 116.16, 119.45, 132.90, 145.57, 145.87, 145.95, 155.73, 156.11, 157.77. HRFABMS calcd for $\mathrm{C}_{30} \mathrm{H}_{26} \mathrm{O}_{12} \mathrm{Na}[\mathrm{M}+\mathrm{Na}]^{+} ;$601.1322; found 601.1263. ${ }^{6 \mathrm{a}, 14}$

Procyanidin B2 (2). In the same manner as prepared 1, compound 13 (40 mg, $0.031 \mathrm{mmol}$ ) gave 2 (11 mg, 58\%) as a colorless solid. Mp 194.5-195.0 ${ }^{\circ} \mathrm{C}$ (decomp.); $[\alpha]_{\mathrm{D}}{ }^{20}+29.3$ (c 0.16, EtOH); ${ }^{1} \mathrm{H}$ NMR (500 $\left.\mathrm{MHz}, \mathrm{CD}_{3} \mathrm{OD}\right): \delta=2.71-2.81(1 \mathrm{H}, \mathrm{m}), 2.84-2.93(1 \mathrm{H}, \mathrm{m}), 3.91(1 \mathrm{H}, \mathrm{m}), 4.09-4.27(1 \mathrm{H}, \mathrm{m}), 4.62(1 \mathrm{H}$, br. s), $4.93(1 \mathrm{H}, \mathrm{m}), 5.05(1 \mathrm{H}, \mathrm{m}), 5.91-5.94(3 \mathrm{H}, \mathrm{m}), 6.70-6.81(4 \mathrm{H}, \mathrm{m}), 6.89(1 \mathrm{H}$, br. s), $7.09(1 \mathrm{H}$, br. s); ${ }^{13} \mathrm{C}-\mathrm{NMR}\left(125 \mathrm{MHz}, \mathrm{CD}_{3} \mathrm{OD}\right): \delta=29.29,29.74,37.21,67.05,67.54,73.56,77.16,79.03,79.29$, 79.55, 95.98, 96.23, 96.50, 100.15, 100.63, 115.34, 115.39, 116.00, 119.47, 127.34, 132.13, 132.34, 145.68, 145.84, 145.93, 146.00, 156.55, 157.42, 157.74, 158.04. HRFABMS calcd for $\mathrm{C}_{30} \mathrm{H}_{26} \mathrm{O}_{12} \mathrm{Na}$ $[\mathrm{M}+\mathrm{Na}]^{+} ; 601.1322$; found $601.1299 .^{4 \mathrm{~d}, 14}$

Procyanidin B3 (3). In the same manner as prepared 1, compound 14 (169 mg) gave 3 (60 mg, 58\%) as a colorless solid. Mp 218-219 ${ }^{\circ} \mathrm{C}$ (decomp.); $[\alpha]_{\mathrm{D}}{ }^{27}-181$ (c 0.29, EtOH); ${ }^{1} \mathrm{H}$ NMR (500 MHz, CD ${ }_{3} \mathrm{OD}$, 0.67:0.33 mixture of rotational isomers) $: \delta=2.49(0.67 \mathrm{H}, \mathrm{dd}, J=8.0,16.2 \mathrm{~Hz}), 2.59(0.33 \mathrm{H}, \mathrm{dd}, J=7.4$, $16.1 \mathrm{~Hz}), 2.76(0.67 \mathrm{H}$, dd, $J=5.5,16.2 \mathrm{~Hz}), 2.82(0.33 \mathrm{H}$, dd, $J=5.6,16.1 \mathrm{~Hz}), 3.78(0.67 \mathrm{H}, \mathrm{m}), 4.08$ $(0.33 \mathrm{H}, \mathrm{m}), 4.26$ (1H, d, $J=9.7 \mathrm{~Hz}), 4.35$ (1H, dd, $J=7.9,9.6 \mathrm{~Hz}), 4.41(1 \mathrm{H}, \mathrm{d}, J=7.8 \mathrm{~Hz}), 4.54(0.67 \mathrm{H}$, d, $J=7.3 \mathrm{~Hz}), 4.75(0.33 \mathrm{H}, \mathrm{d}, J=7.2 \mathrm{~Hz}), 5.79(0.67 \mathrm{H}, \mathrm{d}, J=2.4 \mathrm{~Hz}), 5.82(0.33 \mathrm{H}, \mathrm{d}, J=2.4 \mathrm{~Hz}), 5.85$ 
(0.33H, $J=2.3 \mathrm{~Hz}), 5.89$ (0.67H, d, $J=2.4 \mathrm{~Hz}), 5.95$ (0.33H, s), $6.08(0.67 \mathrm{H}, \mathrm{s}), 6.26(0.67 \mathrm{H}, \mathrm{dd}, J=1.8$, $8.2 \mathrm{~Hz}), 6.48$ (0.67H, dd, $J=1.9,8.2 \mathrm{~Hz}), 6.58(0.67 \mathrm{H}, \mathrm{d}, J=1.9 \mathrm{~Hz}), 6.68(1.33 \mathrm{H}, \mathrm{d}, J=8.2 \mathrm{~Hz}), 6.74$ $(0.67 \mathrm{H}, \mathrm{d}, J=1.9 \mathrm{~Hz}), 6.75(0.67 \mathrm{H}, \mathrm{dd}, J=1.9,8.2 \mathrm{~Hz}), 6.78(0.33 \mathrm{H}, \mathrm{dd}, J=8.2,1.9 \mathrm{~Hz}), 6.80(0.33 \mathrm{H}$, dd, $J=8.2,1.9 \mathrm{~Hz}), 6.96(0.67 \mathrm{H}, \mathrm{d}, J=1.9 \mathrm{~Hz}) ;{ }^{13} \mathrm{C}-\mathrm{NMR}\left(125 \mathrm{MHz}, \mathrm{CD}_{3} \mathrm{OD}, 0.71: 0.29\right.$ mixture of rotational isomers) $: \delta=28.51,28.79,38.64,68.63,68.94,73.74,82.50,83.00,83.98,84.14,96.18,96.34$, 96.96, 97.41, 97.64, 100.62, 102.34, 107.25, 107.30, 108.24, 108.40, 115.28, 115.58, 116.01, 116.13, 116.20, 116.28, 116.49, 119.96, 120.23, 120.70, 121.09, 131.93, 132.45, 132.68, 145.51, 145.65, 145.82, 146.17, 146.52, 154.93, 155.11, 155.68, 155.80, 155.90, 155.99, 157.15, 157.31, 157.42, 158.68, 159.94. HRFABMS calcd for $\mathrm{C}_{30} \mathrm{H}_{25} \mathrm{O}_{12}[\mathrm{M}-\mathrm{H}]^{-}$; 577.1346; found 577.1358. ${ }^{4 \mathrm{~b}}$

Procyanidin B4 (4). In the same manner as prepared 1, compound 15 (46 mg, $0.035 \mathrm{mmol}$ ) gave 4 (10 mg, 51\%) as a colorless solid. ${ }^{6 a} \mathrm{Mp} 178.5-179.5{ }^{\circ} \mathrm{C}$ (decomp.); $[\alpha]_{\mathrm{D}}{ }^{19}-177$ (c 0.097, EtOH); ${ }^{1} \mathrm{H}$ NMR (500 MHz, $\mathrm{CD}_{3} \mathrm{OD}, 1: 1$ mixture of rotational isomers) $: \delta=2.70(0.5 \mathrm{H}, \mathrm{dd}, J=2.3,17.1 \mathrm{~Hz}), 2.81-2.95$ (1.5H, m), 4.06 (0.5H, m), 4.23 (0.5H, m), 4.31-4.32 (1H, m), $4.42(0.5 \mathrm{H}, \mathrm{d}, J=9.7 \mathrm{~Hz}), 4.47$ (0.5H, dd, $J=3.0,5.0 \mathrm{~Hz}), 4.57(0.5 \mathrm{H}, \mathrm{dd}, J=7.9,9.6 \mathrm{~Hz}), 4.63(0.5 \mathrm{H}, \mathrm{d}, J=7.9 \mathrm{~Hz}), 4.81(0.5 \mathrm{H}, \mathrm{s}), 4.93(0.5 \mathrm{H}, \mathrm{s})$, $5.80(0.5 \mathrm{H}, \mathrm{d}, J=2.3 \mathrm{~Hz}), 5.85(0.5 \mathrm{H}, \mathrm{d}, J=2.3 \mathrm{~Hz}), 5.90(0.5 \mathrm{H}, J=2.4 \mathrm{~Hz}), 5.94(0.5 \mathrm{H}, \mathrm{d}, J=2.4 \mathrm{~Hz})$, $5.96(0.5 \mathrm{H}, \mathrm{s}), 6.10(0.5 \mathrm{H}, \mathrm{s}), 6.42-6.46(1 \mathrm{H}, \mathrm{m}), 6.62(0.5 \mathrm{H}, \mathrm{d}, J=8.2 \mathrm{~Hz}), 6.67(0.5 \mathrm{H}, \mathrm{d}, J=1.9 \mathrm{~Hz})$, $6.70(0.5 \mathrm{H}, \mathrm{d}, J=1.9 \mathrm{~Hz}), 6.72(0.5 \mathrm{H}, \mathrm{d}, J=8.2 \mathrm{~Hz}), 6.79(1 \mathrm{H}, \mathrm{dd}, J=1.9,8.1 \mathrm{~Hz}), 6.87(1 \mathrm{H}, \mathrm{d}, J=8.1$ $\mathrm{Hz}), 6.99(0.5 \mathrm{H}, \mathrm{d}, J=1.9 \mathrm{~Hz}), 7.09(0.5 \mathrm{H}, \mathrm{d}, J=1.8 \mathrm{~Hz}) ;{ }^{13} \mathrm{C}-\mathrm{NMR}\left(125 \mathrm{MHz}, \mathrm{CD}_{3} \mathrm{OD}, 1: 1 \mathrm{mixture}\right.$ of rotational isomers) $: \delta=29.42,30.12$, 38.83, 38.91, 67.45, 67.85, 73.86, 79.98, 80.11, 83.93, 84.10, 96.25, 96.54, 97.24, 97.69, 97.80, 99.59, 101.59, 107.22, 107.46, 108.32, 108.76, 114.90, 115.35, 116.02, 116.05, 116.13, 116.39, 116.51, 119.23, 120.32, 120.56, 121.25, 131.78, 132.35, 132.50, 132.65, 145.64, 145.71, 146.01, 146.18, 146.52, 155.43, 155.87, 155.94, 156.38, 156.45, 157.25, 157.33, 157.40, 157.57, 158.60, 158.75. HRFABMS calcd for $\mathrm{C}_{30} \mathrm{H}_{26} \mathrm{O}_{12} \mathrm{Na}[\mathrm{M}+\mathrm{Na}]^{+}$; 601.1322; found 601.1367. ${ }^{\text {6a }}$

Food materials. The apple juice was made from "Fuji (Malus domestica)" apples. The fresh was cut into small pieces, frozen in liquid $\mathrm{N}_{2}$ and freeze-dried. Then, the samples were ground to powdered from using a mixer and stored in a desiccator for further use.

Preparation of fruit phenolic fraction. Before the extraction of phenolics, the freeze-dried flesh powder (10 g) was mixed with petroleum ether in a beaker, stirred and filtered through filter paper on a Büchner funnel to remove lipids (100 $\mathrm{mL} \times 5$ times). The phenolics were then extracted from the residue with $60 \%$ $(\mathrm{v} / \mathrm{v})$ aqueous acetone $(100 \mathrm{~mL} \times 2$ times $)$ in the same manner. The $60 \%$ acetone solution was evaporated until all the organic solvent was removed. The aqueous solution of the extracts was applied onto a 
Sep-Pak Vac 20 cc (5 g) C18 cartridge column which was preconditioned with MeOH (10 mL) and 0.1\% (v/v) TFA in water. The column was washed with $0.1 \%$ TFA solution $(40 \mathrm{~mL})$ and phenolics were eluted with $\mathrm{MeOH}(20 \mathrm{~mL})$. The methanol solution was added to water and evaporated, and the resultant aqueous solution was frozen and then freeze-dried to obtain semi-purified phenolic powder. It was analyzed using HPLC for evaluation of phenolic composition.

HPLC analysis of procyanidin B1-B4 (1-4). Chromatographic separation was carried out on a Luna $5 \mu$ C18 column (150 × 4.6 mm, Phenomenex, Inc., Torrance, CA., USA) with a security guard cartridge (4 $\times 4.6 \mathrm{~mm}$ ) at $40^{\circ} \mathrm{C}$. Solvents were $0.1 \%$ trifluoroacetic acid (A) and $0.1 \%$ trifluoroacetic acid in acetonitrile (B). The gradient program began with 5\% B and was changed to obtain 15\% B at 30 min, $32 \% \mathrm{~B}$ at $35 \mathrm{~min}, 40 \% \mathrm{~B}$ at $45 \mathrm{~min}$, and $75 \% \mathrm{~B}$ at $50 \mathrm{~min}$. The $75 \% \mathrm{~B}$ was maintained until $65 \mathrm{~min}$. The flow rate was $1.0 \mathrm{~mL} / \mathrm{min}$ and the injection volume was $20 \mu \mathrm{L}$. Detection was performed at $280 \mathrm{~nm}$.

\section{ACKNOWLEDGEMENTS}

We thank Geol Cosmetics. Co., LTD for financial support.

\section{REFERENCES AND NOTES}

1. D. Ferreira and X. -C. Li, Nat. Prod. Rep., 2000, 17, 193.

2. D. Ferreira and X. -C. Li, Nat. Prod. Rep., 2002, 19, 517.

3. R. Corder, W. Muller, N. Q. Khan, S. C. Marks, E. G. Wood, M. J. Carrier, A. Crozier, Nature, 2006, 444, 566.

4. (a) W. Tückmantel, A. P. Kozikowski, and L. J. Romanczyk, Jr., J. Am. Chem. Soc., 1999, 121, 12073. (b) A. Saito, N. Nakajima, A. Tanaka, and M. Ubukata, Tetrahedron, 2002, 58, 7829. (c) A. P. Kozikowski, W. Tückmantel, G. Böttcher, and L. J. Romanczyk, Jr., J. Org. Chem., 2003, 68, 1641. (d) A. Saito, N. Nakajima, N. Matsuura, A. Tanaka, and M. Ubukata, Heterocycles, 2004, 62, 479. (e) K. -I. Oyama, M. Kuwano, M. Ito, K. Yoshida, and T. Kondo, Tetrahedron Lett., 2008, 49, 3176.

5. A. P. Kozikowski, W. Tückmantel, and Y. Hu, J. Org. Chem., 2001, 66, 1287.

6. (a) A. Saito, N. Nakajima, A. Tanaka, and M. Ubukata, Heterocycles, 2003, 61, 287. (b) A. Saito, N. Nakajima, A. Tanaka, and M. Ubukata, Tetrahedron Lett., 2003, 44, 5449.

7. I. Tarascou, K. Barathieu, Y. Andé, I. Pianet, E. J. Dufourc, and E. Fouquet, Eur. J Org. Chem., 2006, 5367.

8. For preliminary communication: Y. Mohri, M. Sagehashi, T. Yamada, Y. Hattori, K. Morimura, T. 
Kamo, M. Hirota, and H. Makabe, Tetrahedron Lett., 2007, 48, 5891.

9. H. Kawamoto, F. Nakatsubo, and K. Murakami, Mokuzai Gakkaishi, 1991, 37, 488.

10. A. Saito, N. Nakajima, A. Tanaka, and M. Ubukata, Biosci. Biotechnol. Biochem., 2002, 66, 1764.

11. Recently, Westhuizen and co-workers reported $\mathrm{AgBF}_{4}$ mediated oxidative condensation of tetra- $O$-methyl-3-oxo-catechin with tetra- $O$-methylcatechin: A. C. Matthew, S. L. Bonnet, and J. H. van der Westhuizen, Org. Lett., 2008, 10, 3865.

12. K. Ohomori, N. Ushimaru, and K. Suzuki, Proc. Nat. Acad. Sci. USA, 2004, 101, 12002.

13. C. J. Hayes, B. P. Whittaker, S. A. Watson, and A. M. Grabowska, J. Org. Chem., 2006, 71, 9701.

14. The ${ }^{13} \mathrm{C}-\mathrm{NMR}$ data of 1 and 2 were not described in ref. $4 \mathrm{~d}$ and $6 \mathrm{a}$. It may due to the broadening of the signals.

15. Y. Hamauzu, M. Irie, M. Kondo, and T. Fujita, Food Chem., 2008, 108, 488.

16. B.-J. Xie and co-workers reported that procyanidin B2 was also identified as a major constituent of procyanidin dimer by HPLC analysis in Granny Smith apple: J.-S. Xiao, L. Liu, H. Wu, B.-J. Xie, E.-N. Yang, and Z.-D. Sun, J. Agric. Food Chem., 2008, 56, 2096. 